Journal of

\section{Applied Crystallography}

ISSN 0021-8898

Received 24 November 2005

Accepted 2 April 2006

\title{
Si wafers having one- and two-dimensionally curved (111) planes examined by $\mathrm{X}$-ray diffraction
}

\author{
Hiroshi Okuda, ${ }^{a *}$ Kazuo Nakajima, ${ }^{\mathrm{b}}$ Kozo Fujiwara $^{\mathrm{b}}$ and Shojiro Ochiai ${ }^{\mathrm{a}}$ \\ anternational Innovation Center, Kyoto University, Yoshida, Kyoto 606-8501, Japan, and ${ }^{\mathbf{b}}$ Institute of Materials \\ Research, Tohoku University, Sendai, Japan. Correspondence e-mail: okuda@iic.kyoto-u.ac.jp
}

Si (111) wafers deformed at elevated temperatures into cylindrical or hemispherical shapes have been examined by X-ray diffraction. Well defined 333 peaks shifted with in-plane distance from the centre of the wafer as determined by $\omega$ scans. The shift of the peak position agreed with the curvature of the Si wafer, suggesting that the $\mathrm{Si}$ (111) lattice plane can be designed on an arbitrarily curved surface by this method. These crystals have potential for innovative applications in X-ray instrumentation, for both diffraction/scattering and spectroscopy.

(C) 2006 International Union of Crystallography Printed in Great Britain - all rights reserved crystals with bending strain exceeding the elastic limit can be produced. In the present communication, X-ray diffraction results for Si (111) wafers deformed to either a cylindrical or hemispherical shape by hot plastic pressing are presented. $\mathrm{X}$-ray beam from a conventional X-ray generator has long been one of the most important challenges in X-ray instrumentation. Johann (1931) demonstrated a focusing curved monochromator, and Johansson (1933) proposed an advanced design of a curved crystal having the crystal surface exactly on the focusing circle. The application of these crystals has been limited, partly because of the technical difficulty in deforming a single-crystalline material satisfying two conditions at the same time, i.e. good crystalline quality [full width at half-maximum (FWHM) of the Bragg peak] and a large angle of acceptance. The fabrication of a high-performance curved monochromator has been attempted from two approaches. One is to use nearly perfect crystals, e.g. Si or Ge wafers within their elastic limit, which are fixed by bonding techniques (Sakurai et al., 2002; Collart et al., 2005), and the other is slow deformation of rather soft crystals, e.g. LiF (Morita et al., 1983), having relatively large FWHM values of the Bragg peaks. From the viewpoint of the quality of the diffracted beam, i.e. the resolution of an analyser, the use of nearly perfect crystals such as Si or Ge is essential (Sourtti et al., 1986). Elastic deformation of Si or Ge crystals into a concave shape has the merit that crystal quality should be far better than those deformed plastically, whereas plastic deformation has been, in turn, intentionally used to obtain imperfect crystals to increase allowance for the acceptance of the incident beam (Alianelli et al., 2004). Since the maximum bending strain at the surface is proportional to the thickness and the curvature of a crystal, it is difficult to obtain a thick crystal with a large curvature by elastic deformation. This is a problem with respect to realisation of accurate point-focusing optics, since a Johansson's monochromator requires a crystal whose thickness increases rapidly with the distance from the centre of the crystal (Johansson, 1933). Therefore, plastic deformation of Si is an attractive candidate to realise a focusing monochomator with a large curvature or with a thick crystal, although maintaining sufficient crystal quality during deformation is still a challenging problem. We have demonstrated (Nakajima, Fujiwara, Pan \& Okuda, 2005) that $\mathrm{Si}$ single-crystal wafers can be deformed into a hemispherical shape with a radius as small as $100 \mathrm{~mm}$ without loosing the crystallographic orientation with respect to the crystal surface, and with an acceptably good quality of the Bragg peaks. This means that concave Si single

\section{Experimental}

Si (111) wafers with $0.3 \mathrm{~mm}$ thickness were plastically deformed at high temperatures ranging from $1473 \mathrm{~K}$ to $1623 \mathrm{~K}$ in a furnace under Ar atmosphere (Nakajima et al., 2004; Nakajima, Ohdaira, Fujiwara \& Pan, 2005). The crystals were deformed into a cylindrical or hemispherical shape. The designed curvatures examined in the present work are listed in Table 1. The curvature of the (111) lattice

$\mathrm{Cu} K \alpha_{1}$ radiation was selected using a channel-cut $\mathrm{Ge} 220$ incident monochromator, and shaped to $0.1 \times 1 \mathrm{~mm}$ by entrance slits. The shift in the angle in the $\omega$ scan was measured as a function of the distance of in-plane translation from the centre of the wafer, $x$. The peak shift was measured along two directions perpendicular to each other for each sample, shown as direction $A$ and $B$ in the following figures. The directions for hemispherical crystals were taken to be arbitrary. For cylindrical ones, direction $A$ corresponds to the bending direction, having the designed curvature, and direction $B$ is perpendicular to it.

\section{Results and discussion}

Fig. 1 shows the shift of the peak position for the hemispherically and cylindrically deformed samples with radius $R=100 \mathrm{~mm}$ as a function of $x$. The two directions are shown as 'dir. A' and 'dir. B' in the figure. As shown in Fig. 1(a), the peak position and the FWHM value were determined by a Gaussian fit of the peak. The peak position of the sample changes smoothly as a function of distance for a very wide range of angles of about $15^{\circ}$ for both hemispherical (both directions) and cylindrical (direction $A$ ) deformations. It is also seen that the

Table 1

Curvatures of the samples examined in the experiment.

\begin{tabular}{llll}
\hline $\begin{array}{l}\text { Hemisphere } \\
\text { Cylinder }\end{array}$ & $40 \mathrm{~mm}$ & $50 \mathrm{~mm}$ & $100 \mathrm{~mm}$ \\
& & $100 \mathrm{~mm}$ & $600 \mathrm{~mm}$ \\
\hline
\end{tabular}
plane was evaluated on the basis of 333 diffraction peaks. 


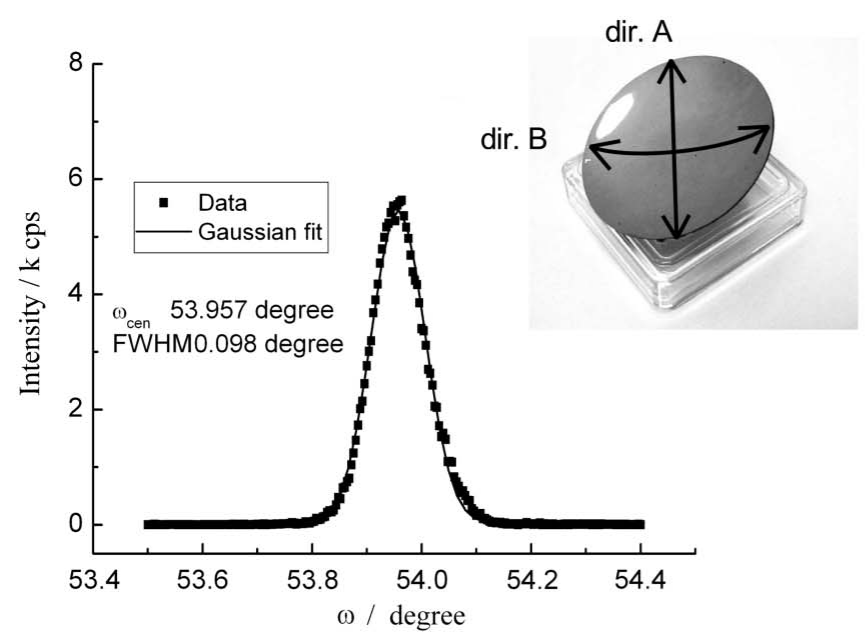

(a)

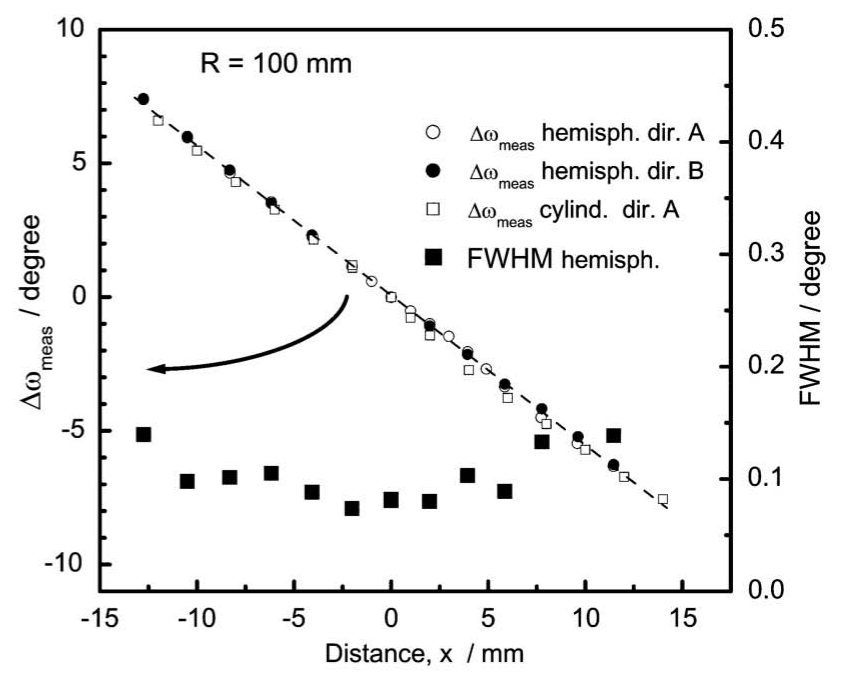

(b)

Figure 1

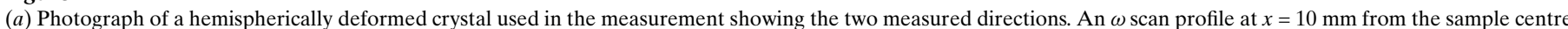

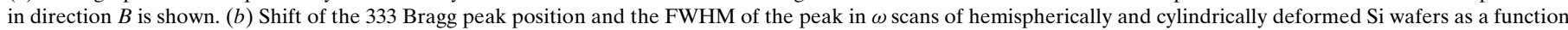
of translation distance of the sample, $x$.

peak shifts for the two directions agree with each other for the hemispherical sample, meaning that the lattice plane curvature of the sample is uniform. The FWHM values of the 333 peak for the sphere are slightly larger near the sample edge, but remain within $0.15^{\circ}$, as shown in the figure. Since the slit width resolution is less than half the measured FWHM, we can conclude that the present FWHM resulted from dislocations introduced by plastic deformation.

The relationship between the measured 333 peak shifts, $\Delta \omega_{\text {meas }}$, and the expected peak shifts, $\Delta \omega_{\text {calc }}$, calculated from the designed surface curvature, $R$, is shown in Fig. 2. The expected peak shift was estimated, with a height correction, by

$$
\Delta \omega_{\text {calc }}=\tan ^{-1}\left[x-h \tan \left(\theta_{333}\right)\right]
$$

with

$$
h=R\left[1-\cos \left(\Delta \omega_{\text {meas }}\right)\right],
$$

where $h$ is the height of the crystal surface at $x \mathrm{~mm}$ from the centre of the crystal, $\theta_{333}$ is the Bragg angle of the 333 diffraction for $\mathrm{Si}$, and $R$ is the radius of curvature of the crystal.

The measured and expected peak shifts agree well for the wafers having different curvatures and in the two directions perpendicular to each other, resulting in a solid angle of more than $0.2 \mathrm{sr}$. These results mean that the hemispherical wafers prepared by the present hot plastic deformation have a well defined (111) lattice plane along the curved surface.

When we examine the deviation of the 333 peak position from the calculated one, the standard deviation for a hemisphere $\left(0.096^{\circ}\right)$ is smaller than that for a cylinder in the curved direction $\left(0.18^{\circ}\right)$ between the wafers deformed to the same radius of $R=100 \mathrm{~mm}$. This might be explained by compatibility of deformation symmetry and slip plane symmetry, but is left for further examination from the viewpoint of the development of dislocation microstructures during hot deformation.

As shown in Figs. 1 and 2, the Si (111) wafers deformed at high temperatures have a well defined (111) lattice plane along the curved surface. Therefore, the sample is in the condition that the singlecrystalline state is maintained by the introduction of uniform dislocations that accommodate the internal strain of the curvature. This implies that the lower limit of the defect density for the complete

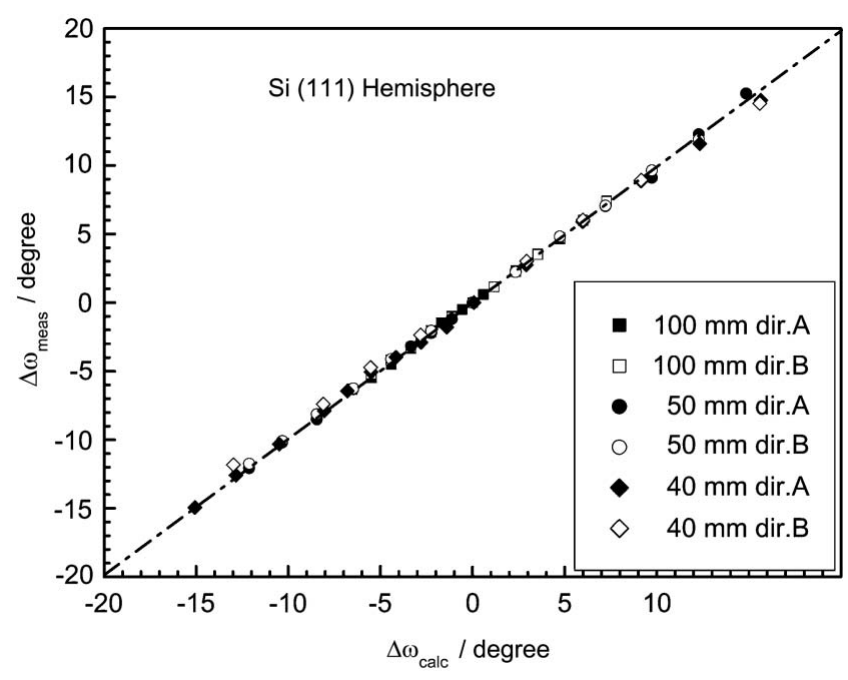

Figure 2

Relationship between the measured peak shift and the calculated peak shift for hemispherical Si wafers deformed to various radii of curvature.

plastic case might be determined by the curvature of the crystal. For example, if a wafer has a radius of curvature of $50 \mathrm{~mm}$, then the dislocation density required to accommodate the curvature of the lattice plane should be of the order of $10^{7} \mathrm{~cm}^{-2}$, which is close to the level of $\mathrm{GaAs} / \mathrm{Si}$, and is rather high for a semiconducting wafer for electronic circuits, and quite high for precise dynamical X-ray optics, which are usually realised using perfect single crystals. However, since the strain is released at the surface when the deformation is perfectly plastic, there may be a further possibility of improving the crystal quality by pseudomorphic regrowth at the surface. This is important since the crystal quality within the penetration depth of $\mathrm{X}$-ray is the most critical issue for X-ray applications.

We have demonstrated that single-crystalline Si (111) wafers deformed at elevated temperatures have (111) lattice planes along their one- or two-dimensionally curved surfaces. This technique allows us to produce novel focusing optics in X-ray and related 
crystallography, and offers the potential to improve the efficiency of $\mathrm{X}$-ray usage in divergent sources such as conventional generators, by a magnitude of 10 to $10^{3}$. Related optical components and instruments (Nakajima, Okuda \& Fujiwara, 2005) are now under development.

KN acknowledges the financial support through a Grant-in-Aid for Scientific Research from MEXT, Japan, under proposal number 17656002. HO acknowledges financial aid from the Institute of Materials Research, Tohoku University, for the joint research in 2005 .

\section{References}

Alianelli, L., Sanchez del Rio, M. \& Felici, R. (2004). J. Appl. Cryst. 37, 732742.

Collart, E., Shukla, A., Gelebart, F., Morand, M., Malgrange, C., Bardou, N., Madouri, A. \& Pelouard, J. (2005). J. Synchrotron Rad. 12, 473-478.

Johann, H. H. (1931). Z. Phys. 69, 185-206.

Johansson, T. (1933). Z. Phys. 71, 507-528.

Morita, S., Kadota, K. \& Fujita, J. (1983). Jpn J. Appl. Phys. 22, 1283-1289.

Nakajima, K., Fujiwara, K. \& Pan, W. (2004). Appl. Phys. Lett. 85, 5896-5898.

Nakajima, K., Fujiwara, K., Pan, W. \& Okuda, H. (2005). Nat. Mater. 4, 47-49.

Nakajima, K., Ohdaira, K., Fujiwara, K. \& Pan, W. (2005). Solar Energy Mater. Solar Cells, 88, 323-329.

Nakajima, K., Okuda, H. \& Fujiwara, K. (2005). Intern. Patent. Appl. PCT/ JP2005/15442.

Sakurai, K., Eba, H., Inoue, K. \& Yagi, N. (2002). Anal. Chem. 74, 4532-4535. Sourtti, P., Patteison, P. \& Weyrich, W. (1986) J. Appl. Cryst. 19, 343-352. 